\title{
Prognostic Parameters on Baseline and Interim F-18-FDG-PET/CT in Diffuse Large B-cell Lymphoma Patients
}

Sándor Czibor ( $\nabla$ czibor.sandor@med.semmelweis-univ.hu )

Semmelweis University; Medical Imaging Centre, Department of Nuclear Medicine https://orcid.org/0000-0002-7679-3137

Robert Carr

King's College London, Guy's \& St. Thomas' Hospital, Department of Hematology

Francisca Redondo

Instituto Oncologico Fundacion Arturo Lopez Perez

Charity Gorospe

Saint Luke's Medical Center

Chirayu Auewarakul

HRH Princess Chulabhorn College of Medical Science

Juliano Cerci

Cuanta Diagnóstico e Terapia

Diana Paez

International Atomic Energy Agency; Department of Nuclear Sciences and Application, Division of Human Health, Nuclear Medicine and Diagnostic Imaging Section

Stefano Fanti

IRCCS University Hospital of Bologna S Orsola-Malpighi Polyclinic: Azienda Ospedaliero-Universitaria di Bologna Policlinico Sant'Orsola-Malpighi Tamás Györke

Semmelweis University; Medical Imaging Centre, Department of Nuclear Medicine

\section{Research Article}

Keywords: Positron emission tomography, Diffuse large B-cell lymphoma, Metabolic tumour volume, Deauville score, rPET

Posted Date: October 8th, 2021

DOI: https://doi.org/10.21203/rs.3.rs-958512/v1

License: (c) (1) This work is licensed under a Creative Commons Attribution 4.0 International License. Read Full License 


\section{Abstract}

Purpose FDG-PET/CT is a widely used imaging method in the management of diffuse large B-cell lymphomas (DLBCL). Our aim was to investigate the prognostic performance of different PET-biomarkers in a multicentre setting.

Methods We investigated baseline volumetric values (MTV and TLG, also normalized for body weight) segmented with three different methods (>SUV4 [glob4]; $41 \%$ isocontour [ $41 \mathrm{pc}$ ], and a gradiant-based lesion growing algorithm [grad]) and interim parameters (Deauville-score, $\triangle \mathrm{SUVmax}, \mathrm{modified} \mathrm{qPET}$, and rPET) alongside clinical parameters (stage, R-IPI), using 24-month progression-free survival as the clinical endpoint. Receiver operating characteristics analyses were performed to define optimal cut-off points for the continous PET-parameters.

Results 107 DLBCL patients were included (54 women; mean age: 53.7 years). MTV and TLG calculations showed good correlation among glob4, 41 pc and grad methods, however, optimal cut-off points were markedly different.

Significantly different PFS was observed between low- and high-risk groups according to baseline MTV, bwaMTV, TLG, bwaTLG, as well as interim parameters Deauville-score, $\triangle$ SUVmax, mqPET, and rPET. Univariate Cox-regression analyses showed hazard ratios lowest for bwaMTVglob4 (HR=2.3) and highest for rPET (HR=9.09). In a multivariate Cox-regression model, rPET was shown to be an independent predictor of PFS ( $p=0.041 ; \mathrm{HR}=9.15)$. A combined analysis showed that $\triangle$ SUVmax positive patients with high MTV formed a group with distinctly poor PFS (35.3\%).

Conclusion Baseline MTV and TLG values and optimal cut-off points achieved with different segmentation methods varied markedly and showed limited prognostic impact. Interim PET/CT parameters provided more accurate prognostic information with semiquantitative „Deauville-like” parameters performing best in the present study.

\section{Introduction}

Diffuse Large B-cell Lymphoma (DLBCL) is a clinically, pathologically, and molecularly heterogenous haematological malignancy, considered the most common subtype of non-Hodgkin lymphomas [1]. In its initial clinical staging, the utility of F-18-fluoro-deoxy-glucose (FDG) positron emission tomography/computed tomography (PET/CT) examination has gained vast evidence and is incorporated in current recommendations [2].

Aside from well-researched clinical, pathological and molecular prognostic factors, several F-18-FDG-PET/CT-based biomarkers have emerged in the last decade, also carrying prognostic information (beyond its inherent prognostic value in defining the clinical stage of DLBCL).

Of these parameters, metabolic tumour volume (MTV) and total lesion glycolysis (TLG) have shown promise to yield added prognostic value to established clinical scores, e.g. the Interational Prognostic Index (IPI) and its modifications, the revised IPI (R-IPI) and the National Comprehensive Cancer Network-IPI (NCCN-IPI) [3-8].

Beyond its utility as baseline investigation, FDG-PET/CT plays an important role in the evaluation of treatment response at the end of therapy, or even in an early assessment, interim setting. Robust and wide-spread evaluation criteria based on the Deauville-5-point-scale have been established to decide the presence or absence of complete metabolic remission $[9,10]$. Aside from the ordinal Deauville-score (DS), continuous values have been investigated in highgrade lymphomas, most notably the proportianal decrease of lesion maximal standardized uptake value (SUVmax) and, to a lesser extent, semiquantitative „Deauville-like” parameters, such as qPET and rPET [11-18].

Our aim was to investigate the prognostic performance of baseline volumetric values (MTV and TLG) and interim parameters (DS and semiquantitative) derived from the FDG-PET/CT scans of DLBCL patients in a multicentre setting.

\section{Methods}

We investigated the baseline and interim PET/CT scans of DLBCL patients included in a multicentric study coordinated by the International Atomic Energy Agency (IAEA) who received R-CHOP (rituximab combined with cyclophosphamide, doxorubicin, vincristine, and prednisolone) immunochemotherapy. The study design was elaborately described before [19], this time a reduced number of patients was included in our sample after the following exclusion criteria: 1) treatment other than R-CHOP; 2) studies performed on a stand-alone PET scanner; 3) studies performed on different PET/CT scanners in baseline and interim setting; 4) missing or compromised imaging data; 5) event-free follow-up lasting less than 24 months. Ten centers in the same number of countries (Brazil, Chile, Hungary, India, Italy, Pakistan, the Philippines, South Korea, Thailand, and Turkey) participated in the IAEA study. The research was approved by the respective ethical review board of each participating centre and all subjects signed an informed consent form.

Clinical stage was determined by the baseline PET/CT scans according to the Lugano criteria and R-IPI was calculated for each patient [2, 7]. Lymphoma lesions on baseline PET images were delineated with three different methods: 1) >SUV4 (glob4); 41\% isocontour VOI around the local maximum point (41pc); a vendor-specific gradiant-based lesion growing algorithm (grad), performed with Mediso InterView Fusion software (Mediso Medical Imaging Systems, Budapest, Hungary). MTV was calculated as the sum of all lymphoma lesions' volume on PET images, and TLG was determined as the sum of the product of each lesion's metabolic volume and SUVmean. Both MTV and TLG values were normalized for patient body weight, thus introdicing bwaMTV and bwaTLG values. Receiver operating characteristics (ROC) analyses were performed to define optimal cut-off points for MTV, TLG, bwaMTV, and bwaTLG for the three different segmentation methods.

Interim PET/CT scans were analyzed visually according to the Deauville criteria, resulting in Deauville-scores (DS) 1-5, and semiquantitatively. Deauville-score 5 was defined as lesion SUVmax three times over liver SUVmax. The semiquantitative evaluation methods included the proportional change in SUVmax in 
percents between the baseline and interim scans ( $\triangle$ SUVmax), and two semiquantitative „Deauville-like” parameters for which a $3 \mathrm{~cm}$ diameter spheric VOI was placed in the unaffected part of the right liver lobe. Modified qPET (mqPET) is the proportion of the hottest lesion's SUVpeak (the SUVmean of the hottest 1

$\mathrm{cm}^{3}$ in the lesion VOI) and the SUVmean of the liver VOI - the original qPET value, described first by Hasenclever et al. in pediatric Hodgkin's lyphoma used the mean SUV of the hottest 4 adjacent voxels in the lesion. Our use of the $1 \mathrm{~cm}^{3}$ SUVpeak was based on the lack of adequate software as well as the hypothesis that in adult patients this volume would not lead to considerable distortion in the results. The ratio PET (rPET), as described before, is the proportion of the SUVmax in the hottest lesion and the liver reference VOI $[16,17]$.

When establishing the diagnostic performance of the above different prognostic biomarkers, 24-month progression-free survival was the clinical endpoint. Statistical calculations were performed in the R environment (The R Foundation, https://www.r-project.org) with R Studio software (RStudio PBC; Boston, MA, USA).

\section{Results}

\section{Patient characteristics}

107 patients were included in the present study (mean age: 53.7; range: 16-83 years) with 53 women and 54 men among them. The majority of patients were from Hungary (57) and Chile (36), while eight, four, and two of them were from Thailand, the Philippines, and Italy, respectively. $58 \%$ of the patients presented with advanced stage disease. Further patient information is provided in Table 1.

Table 1

Patient demographic and clinical data

\begin{tabular}{|c|c|c|}
\hline \multicolumn{2}{|c|}{ Patient characteristics } & \multirow{2}{*}{$\begin{array}{l}n=107(100 \%) \\
54\end{array}$} \\
\hline Sex & Male & \\
\hline & Female & 53 \\
\hline \multirow[t]{3}{*}{ Age } & Range & $16-83$ \\
\hline & Median & 56 \\
\hline & $>60$ years & $44(41 \%)$ \\
\hline \multicolumn{3}{|c|}{ Performance status } \\
\hline & $0-1$ & $83(78 \%)$ \\
\hline & $2-4$ & $24(22 \%)$ \\
\hline \multicolumn{3}{|c|}{ Stage } \\
\hline & I & $16(15 \%)$ \\
\hline & $\|$ & $29(27 \%)$ \\
\hline & III & $19(18 \%)$ \\
\hline & IV & $43(40 \%)$ \\
\hline \multicolumn{3}{|c|}{ R-IPI } \\
\hline & good & $24(22 \%)$ \\
\hline & intermediate & $48(45 \%)$ \\
\hline & poor & $35(33 \%)$ \\
\hline \multicolumn{3}{|c|}{ Timing of interim PET/CT } \\
\hline & after 2 cycles of R-CHOP & $90(84 \%)$ \\
\hline & after 3 cycles of R-CHOP & $17(16 \%)$ \\
\hline
\end{tabular}

\section{Comparison of volumetric parameters achieved by different delineation methods}

MTV and TLG calculations showed good correlation among glob4, 41pc and grad methods (Table 2), despite occasionally resulting in markedly different volumes (Figure 1). ROC analyses yielded markedly different optimal cut-off points for MTV, TLG, bwaMTV, and bwaTLG with the three different segmentation methods (Table 3). Areas under the curve (AUCs) did not show a significant difference between MTV vs. bwaMTV and TLG vs. bwaTLG with the corresponding segmentation methods, the values ranging between 0.62 and 0.68 (Table 3). More diverse values in sensitivity, specificity, positive and negative predictive values, and diagnostic accuracy could be observed, primarily among the same volumetric parameters with different segmentation methods and not between traditional and body weight-adjusted MTV or TLG. 
Table 2

Pearson-correlation coefficients between volumetric

parameters by different segmentation methods

\begin{tabular}{|llllll|}
\hline MTV & $41 p c$ & grad & TLG & $41 p c$ & grad \\
\hline glob4 & 0.872 & 0.849 & glob4 & 0.981 & 0.984 \\
\hline $41 p c$ & & 0.962 & $41 p c$ & & 0.993 \\
\hline
\end{tabular}

Table 3

Cut-off values, areas-under-the-curve (AUCs) and diagnostic performance of volumetric parameters by different segmentation methods

\begin{tabular}{|lllllllllllll|}
\hline & MTV & & \multicolumn{3}{c}{ TLG } & \multicolumn{3}{c}{ bwaMTV } & \multicolumn{3}{c|}{ bwaTLG } \\
\hline & glob4 & $41 \mathrm{pc}$ & grad & glob4 & $41 \mathrm{pc}$ & grad & glob4 & $41 \mathrm{pc}$ & grad & glob4 & $41 \mathrm{pc}$ & grad \\
\hline Cut-off & 122.5 & 257.5 & 334.9 & 714.7 & 1207 & 2112 & 2.55 & 2.68 & 7.84 & 15.5 & 13.2 & 53.3 \\
\hline AUC & 0.63 & 0.65 & 0.66 & 0.62 & 0.64 & 0.65 & 0.65 & 0.67 & 0.68 & 0.63 & 0.65 & 0.65 \\
\hline Sensitivity & $77.8 \%$ & $74.1 \%$ & $63 \%$ & $81.4 \%$ & $77.8 \%$ & $62.9 \%$ & $66.7 \%$ & $77.8 \%$ & $55.6 \%$ & $70.4 \%$ & $77.8 \%$ & $51.9 \%$ \\
\hline Specificity & $47.5 \%$ & $56.3 \%$ & $70 \%$ & $41.3 \%$ & $51.3 \%$ & $62.5 \%$ & $56.3 \%$ & $57 \%$ & $76.2 \%$ & $51.3 \%$ & $48.8 \%$ & $73.8 \%$ \\
\hline PPV & $33.3 \%$ & $36.4 \%$ & $41.4 \%$ & $31.9 \%$ & $35 \%$ & $36.2 \%$ & $34 \%$ & $36.2 \%$ & $44.1 \%$ & $32.8 \%$ & $33.9 \%$ & $40 \%$ \\
\hline NPV & $86.3 \%$ & $86.5 \%$ & $84.8 \%$ & $86.8 \%$ & $87.2 \%$ & $83.3 \%$ & $83.3 \%$ & $89.1 \%$ & $83.6 \%$ & $83.7 \%$ & $86.7 \%$ & $81.9 \%$ \\
\hline Accuracy & $55.1 \%$ & $60.7 \%$ & $68.2 \%$ & $51.4 \%$ & $57.9 \%$ & $62.6 \%$ & $58.9 \%$ & $61.9 \%$ & $71 \%$ & $56.1 \%$ & $56.1 \%$ & $68.2 \%$ \\
\hline
\end{tabular}

\section{Prognostic value of baseline and interim biomarkers}

With the aim of a more transparent data presentation, only the >SUV4-method-based (glob4) volumetric values (MTV, TLG, bwaMTV, bwaTLG) are presented, as it is considered the most easily reproducible segmentation method.

ROC analyses were performed to define optimal cut-off points for I-PET semiquantitative values, yielding values of $-77.22 \%, 1.32$, and 1.54 for $\Delta S$ SUVmax, mqPET, and rPET, respectively. Sensitivity, specificity, positive and negative predictive values, and diagnostic accuracy of interim parameters are detailed in Table 4.

Table 4

Cut-off values and diagnostic performance of interim

\begin{tabular}{|lllll|}
\hline \multicolumn{5}{c}{ parameters } \\
\hline Cut-off & & $-71.22 \%$ & 1.32 & 1.54 \\
\hline Sensitivity & $59.3 \%$ & $48.1 \%$ & $59.2 \%$ & $55.6 \%$ \\
Specificity & $83.8 \%$ & $85 \%$ & $87.5 \%$ & $92.5 \%$ \\
\hline PPV & $55.2 \%$ & $52 \%$ & $61.5 \%$ & $71.4 \%$ \\
\hline NPV & $85.9 \%$ & $82.9 \%$ & $86.4 \%$ & $86 \%$ \\
Accuracy & $77.6 \%$ & $75.7 \%$ & $80.4 \%$ & $83.2 \%$ \\
\hline
\end{tabular}

Progression-free survival in the whole cohort was 75\% (Figure 2). Interestingly, log-rank survival analysis did not show a significant difference between the PFS of early and advanced stage patients ( $82 \%$ vs. $69 \%$ ). Dividing the patients into two groups according to calculated optimal cut-offs or predefined values (in case of DS) resulted in significantly different PFS for baseline MTV, bwaMTV, TLG, bwaTLG, as well as interim parameters DS (1-3 vs. 4-5), $\triangle$ SUVmax, mqPET, and rPET (Table 5, Figure 3).

Table 5

24-months progression-free survival rates of low- and high-risk groups according to different parameters

\begin{tabular}{|lllllllll|}
\hline & MTV & bwaMTV & TLG & bwaTLG & DS & SSUVmax & mqPET & rPET \\
\hline low-risk & $86 \%$ & $83 \%$ & $87 \%$ & $84 \%$ & $86 \%$ & $83 \%$ & $86 \%$ & $86 \%$ \\
\hline high-risk & $67 \%$ & $66 \%$ & $69 \%$ & $67 \%$ & $45 \%$ & $48 \%$ & $38 \%$ & $29 \%$ \\
\hline
\end{tabular}


Univariate Cox-regression analyses showed a significant difference between low- and high-risk groups except for early/advanced stage and low/high bwaTLGglob4, with calculated hazard ratios (HRs) the lowest for bwaMTVglob4 (HR=2.3) and the highest for rPET (HR=9.09) among the remaining prognostic parameters (Table 6). In a multivariate Cox-regression model including DS (1-3 vs. 4-5), $\triangle$ SUVmax, rPET, MTV, and clinical stage (early vs. advanced) only rPET was shown to be a significant independent predictor of PFS ( $p=0.041 ; H R=9.15)$ (Figure 4).

Table 6

Univariate Cox-regression model hazard ratios (HRs) and p-values

\begin{tabular}{|c|c|c|c|c|c|c|c|c|c|c|c|c|c|c|c|c|}
\hline & \multirow{2}{*}{$\begin{array}{l}\text { Clinical } \\
\text { stage }\end{array}$} & \multicolumn{3}{|l|}{ MTV } & \multicolumn{3}{|l|}{ TLG } & \multicolumn{3}{|c|}{ bwaMTV } & \multicolumn{3}{|c|}{ bwaTLG } & \multicolumn{3}{|c|}{ Interim parameters } \\
\hline & & glob4 & $41 \mathrm{pc}$ & grad & glob4 & $41 \mathrm{pc}$ & grad & glob4 & $41 \mathrm{pc}$ & grad & glob4 & $41 \mathrm{pc}$ & grad & DS & $\Delta S U V \max$ & mqPET \\
\hline $\mathrm{HR}$ & 1.8 & 2.7 & 3.1 & 3.3 & 2.7 & 3.1 & 2.5 & 2.4 & 3.4 & 3.4 & 2.2 & 3.2 & 2.7 & 5.7 & 4.1 & 6.7 \\
\hline $\mathrm{p}$ & 0.163 & 0.03 & 0.01 & 0.003 & 0.047 & 0.014 & 0.019 & 0.031 & 0.008 & 0.002 & 0.057 & 0.02 & 0.01 & $<0.001$ & $<0.001$ & $<0.001$ \\
\hline
\end{tabular}

A combined analysis was performed by forming four groups according to low/high MTV and DS 1-3 vs. 4-5. Kaplan-Meier curves showed a good survival rate for DS 1-3 patients and poor PFS for DS 4-5 patients, irrespective of MTV. A similar analysis with $\triangle$ SUVmax and MTV resulted in relatively good PFS for all $\triangle S U V m a x$ negative patients and $\triangle$ SUVmax positive patients with low MTV, while $\triangle$ SUVmax positive patients with high MTV formed a group with distinctly poor PFS where 11 of 17 patients showed progression within two years (Figure 5).

\section{Discussion}

Several different segmentation algorithms have been used to determine baseline MTV in DLBCL patients. llyas at el investigated the SUV $\geq 2.5$, the $41 \%$, and the "PERCIST" ( $\geq 1.5 \times$ mean SUV + 2 standard deviations in a $3 \mathrm{~cm}^{3}$ right liver lobe VOI) methods. The three segmentation methods yielded different optimal cut-off points for predicting PFS, ranging $166-400 \mathrm{~cm}^{3}$ which is similar to our results of $123-345 \mathrm{~cm}^{3}$ [20]. The same tendency can be observed in MTV measurements of solid tumours as shown by Zhuang et al. who performed eight different segmentations in non-smal cell lung cancer patients that yielded significantly different MTV values [21].

Our data indicate that although MTV and TLG yielded only moderately promising prognostic performance and areas-under-the-curve on ROC analyses, the gradient-based segmentation algorithm resulted in the best values, especially in terms of sensitivity and diagnostic accuracy. However, as this latter algorithm is vendor-specific, its wide-spread use might be limited. TLG did not have better prognostic performance than MTV with the corresponding segmentation methods.

Apart from optimal cut-off points varying in the same patient cohort, MTV also shows a sample-dependency as markedly different values can be found among studies performed with the same (or highly similar) segmentation methodology, as in standalone studies referenced in the llyas paper and in a metaanalyses by Xie et al. and Guo et al., with optimal cut-off points ranging between 66 and $601.2 \mathrm{~cm}^{3}$ for the SUV $\geq 2.5$ method and between 16.1 and $550 \mathrm{~cm}$ for the $40-41 \%$ methods $[4,20,22-26]$.

As radiomics become more prevalent in several imaging research fields, standardization is paramount and the authors would recommend and support collaborations similar to the Image Biomarker Standardization Initiative to make PET imaging parameters more reliable and comparable among centres [27]. Still, as a basis of nearly all calculations, SUVs are also highly variable among studies and this points to a limitation of the current multicentric study as devices had not been cross-calibrated. At present, the reproducibility of SUVs can be supported by the implementation of EARL Harmonization Programme, however, our study had been concluded before its introduction [28].

To the authors' best knowledge, it is the first time that body weight-adjusted (bwa) MTV and TLG values are published. The aim behind the introduction of this normalization was to enable a personalized and more accurate measurement of the impact of tumour burden (normalization to body surface area or lean body mass would also be a feasible option, however, our current dataset did not include patient height in all cases thus making such calculations impossible). Despite bwaMTV and bwaTLG not yielding improved prognostic values over MTV and TLG, respectively, there were a selected few cases where body weightadjusted MTV stratified the patient in the correct risk-group as opposed to regular MTV (Figure 6). These values could be further investigated in larger cohorts as their calculation can be easily carried out. Moreover, body surface area could also serve as a parameter for MTV normalization.

$\triangle$ SUVmax as a prognostic factor has gained a wider presence in the literature in recent years, the majority of the studies finding optimal cut-off points around $66 \%$ which our finding of $71.22 \%$ is close to [12]. Interestingly, in our study, $\Delta S U V$ max evaluation did not result in better prognostic values than the visual Deauville-score method in the whole patient cohort.

Semiquantitative „Deauville-like” parameters may be more robust than $\triangle$ SUVmax in a multicentric setting as the variability in SUVs is at least partially mitigated by using ratios with a reference region. Neither qPET nor rPET values have an extensive literature in DLBCL, especially not in multicentric studies [13-18]. The optimal cut-off for mqPET was 1.32 in our DLBCL cohort which is highly similar to the established qPET cut-off in pediatric Hodgkin's lymphoma patiens based on a 4-voxel-SUVpeak. The optimal cut-off for rPET of 1.54 was higher than the 1.14 and 1.4 values published by Annunziata et al. and Toledano et al., respectively, and close to Fan and coworkers' finding of 1.6 [16-18]. In our study, both mqPET and rPET evaluation yielded moderately more accurate prognostic results than DS stratification.

Interim parameters had a higher hazard ratio in univariate Cox-regression analyses than baseline volumetric parameters while multivariate Cox-regression analysis resulted in rPET as the only independent predictor of PFS. Also, combined analyses showed that good early treatment response (i.e. DS 1-3) has a

Page 5/12 
higher impact on PFS than baseline MTV. This finding is contradictory to that of published by Mikhaeel et al. who found that patients with MTV $\geq 400 \mathrm{~cm}{ }^{3}$ had a worse prognosis, irrespective of DS on interim scans [2]. Furthermore, in the present study the combination of baseline MTV and $\triangle S$ SUVmax enabled to define a group with particularly poor prognosis (i.e. patients with high baseline MTV and DS4-5 on interim scan).

\section{Conclusion}

Baseline MTV values and optimal cut-off points achieved with different segmentation methods varied markedly and showed limited prognostic impact. Interim PET/CT parameters provided more accurate prognostic information with semiquantitative „Deauville-like” parameters (mqPET and rPET) performing best in the present study. A combination of baseline MTV and $\triangle$ SUVmax allowed to separate a patient group with particularly poor prognosis.

\section{Declarations}

\section{Funding}

The study was funded and supported by the International Atomic Energy Agency (Coordinated Research Project E1.50.20).

\section{Compliance with ethical standards}

\section{Conflict of interest}

The authors declare that they have no conflict of interest.

\section{Ethics approval}

All procedures performed in studies involving human participants were in accordance with the ethical standards of the institutional and/or national research committee and with the 1964 Helsinki declaration and its later amendments or comparable ethical standards.

\section{Informed consent}

Informed consent was obtained from all individual participants included in the study.

\section{Availability of data and material}

The datasets generated during and/or analyzed during the current study are available from the corresponding author on reasonable request.

\section{Authors' contributions}

All authors contributed to the material preparation, data collection and analysis. The first draft of the manuscript was written by Sándor Czibor and all authors commented on previous versions of the manuscript. All authors read and approved the final manuscript.

\section{Acknowledgments}

We would like to thank all the patients for their participation in the research. We are grateful to the involved medical staff, alongside the International Atomic Energy Agency for their efforts to make this study possible. We thank László Szakács for his contributions to the statistical analysis.

\section{References}

1. Li S, Young KH, Medeiros LJ. Diffuse large B-cell lymphoma. Pathology. 2018;50:74-87. doi:10.1016/j.pathol.2017.09.006.

2. Cheson BD, Fisher RI, Barrington SF, Cavalli F, Schwartz LH, Zucca E, et al. Recommendations for initial evaluation, staging, and response assessment of Hodgkin and non-Hodgkin lymphoma: the Lugano classification. J Clin Oncol. 2014;32:3059-68. doi:10.1200/JC0.2013.54.8800.

3. Xie M, Zhai W, Cheng S, Zhang H, Xie Y, He W. Predictive value of F-18 FDG PET/CT quantization parameters for progression-free survival in patients with diffuse large B-cell lymphoma. Hematology. 2016;21:99-105. doi:10.1179/1607845415Y.0000000033.

4. Xie M, Wu K, Liu Y, Jiang Q, Xie Y. Predictive value of F-18 FDG PET/CT quantization parameters in diffuse large B cell lymphoma: a meta-analysis with 702 participants. Med Oncol. 2015;32:446. doi:10.1007/s12032-014-0446-1.

5. Islam P, Goldstein J, Flowers CR. PET-derived tumor metrics predict DLBCL response and progression-free survival. Leuk Lymphoma. 2019;60:1965-71. doi:10.1080/10428194.2018.1562181.

6. Project IN-HsLPF. A predictive model for aggressive non-Hodgkin's lymphoma. N Engl J Med. 1993;329:987-94. doi:10.1056/NEJM199309303291402.

7. Sehn LH, Berry B, Chhanabhai M, Fitzgerald C, Gill K, Hoskins P, et al. The revised International Prognostic Index (R-IPI) is a better predictor of outcome than the standard IPI for patients with diffuse large B-cell lymphoma treated with R-CHOP. Blood. 2007;109:1857-61. doi:10.1182/blood-2006-08-038257.

8. Zhou Z, Sehn LH, Rademaker AW, Gordon LI, Lacasce AS, Crosby-Thompson A, et al. An enhanced International Prognostic Index (NCCN-IPI) for patients with diffuse large B-cell lymphoma treated in the rituximab era. Blood. 2014;123:837-42. doi:10.1182/blood-2013-09-524108.

9. Meignan M, Gallamini A, Haioun C. Report on the First International Workshop on Interim-PET-Scan in Lymphoma. Leuk Lymphoma. 2009;50:1257-60. doi:10.1080/10428190903040048. 
10. Barrington SF, Mikhaeel NG, Kostakoglu L, Meignan M, Hutchings M, Müeller SP, et al. Role of imaging in the staging and response assessment of lymphoma: consensus of the International Conference on Malignant Lymphomas Imaging Working Group. J Clin Oncol. 2014;32:3048-58.

doi:10.1200/JC0.2013.53.5229.

11. Lin C, Itti E, Haioun C, Petegnief Y, Luciani A, Dupuis J, et al. Early 18F-FDG PET for prediction of prognosis in patients with diffuse large B-cell lymphoma: SUV-based assessment versus visual analysis. J Nucl Med. 2007;48:1626-32. doi:10.2967/jnumed.107.042093.

12. Itti E, Meignan M, Berriolo-Riedinger A, Biggi A, Cashen AF, Véra P, et al. An international confirmatory study of the prognostic value of early PET/CT in diffuse large B-cell lymphoma: comparison between Deauville criteria and $\Delta$ SUVmax. Eur J Nucl Med Mol Imaging. 2013;40:1312-20. doi:10.1007/s00259-013-2435-6.

13. Hasenclever D, Kurch L, Mauz-Körholz C, Elsner A, Georgi T, Wallace H, et al. qPET - a quantitative extension of the Deauville scale to assess response in interim FDG-PET scans in lymphoma. Eur J Nucl Med Mol Imaging. 2014;41:1301-8. doi:10.1007/s00259-014-2715-9.

14. Kurch L, Hasenclever D, Kluge R, Georgi T, Tchavdarova L, Golombeck M, et al. Only strongly enhanced residual FDG uptake in early response PET (Deauville 5 or qPET $\geq 2$ ) is prognostic in pediatric Hodgkin lymphoma: Results of the GPOH-HD2002 trial. Pediatr Blood Cancer. 2019;66:e27539. doi:10.1002/pbc.27539.

15. Georgi TW, Kurch L, Hasenclever D, Warbey VS, Pike L, Radford J, et al. Quantitative assessment of interim PET in Hodgkin lymphoma: An evaluation of the qPET method in adult patients in the RAPID trial. PLoS One. 2020;15:e0231027. doi:10.1371/journal.pone.0231027.

16. Annunziata S, Cuccaro A, Calcagni ML, Hohaus S, Giordano A, Rufini V. Interim FDG-PET/CT in Hodgkin lymphoma: the prognostic role of the ratio between target lesion and liver SUVmax (rPET). Ann Nucl Med. 2016;30:588-92. doi:10.1007/s12149-016-1092-9.

17. Fan Y, Zhang Y, Yang Z, Ying Z, Zhou N, Liu C, et al. Evaluating early interim fluorine-18 fluorodeoxyglucose positron emission tomography/computed

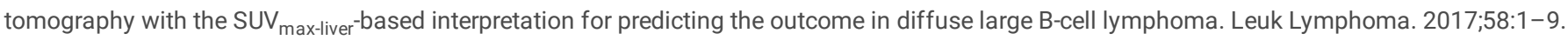
doi:10.1080/10428194.2016.1277384.

18. Toledano MN, Vera P, Tilly H, Jardin F, Becker S. Comparison of therapeutic evaluation criteria in FDG-PET/CT in patients with diffuse large-cell B-cell lymphoma: Prognostic impact of tumor/liver ratio. PLoS One. 2019;14:e0211649. doi:10.1371/journal.pone.0211649.

19. Carr R, Fanti S, Paez D, Cerci J, Györke T, Redondo F, et al. Prospective international cohort study demonstrates inability of interim PET to predict treatment failure in diffuse large B-cell lymphoma. J Nucl Med. 2014;55:1936-44. doi:10.2967/jnumed.114.145326.

20. Ilyas H, Mikhaeel NG, Dunn JT, Rahman F, Møller H, Smith D, et al. Defining the optimal method for measuring baseline metabolic tumour volume in diffuse large B cell lymphoma. Eur J Nucl Med Mol Imaging. 2018;45:1142-54. doi:10.1007/s00259-018-3953-z.

21. Zhuang M, García DV, Kramer GM, Frings V, Smit EF, Dierckx R, et al. Variability and Repeatability of Quantitative Uptake Metrics in. J Nucl Med. 2019;60:600-7. doi:10.2967/jnumed.118.216028.

22. Guo B, Tan X, Ke Q, Cen H. Prognostic value of baseline metabolic tumor volume and total lesion glycolysis in patients with lymphoma: A meta-analysis. PLoS One. 2019;14:e0210224. doi:10.1371/journal.pone.0210224.

23. Sasanelli M, Meignan M, Haioun C, Berriolo-Riedinger A, Casasnovas RO, Biggi A, et al. Pretherapy metabolic tumour volume is an independent predictor of outcome in patients with diffuse large B-cell lymphoma. Eur J Nucl Med Mol Imaging. 2014;41:2017-22. doi:10.1007/s00259-014-2822-7.

24. Chang CC, Cho SF, Chuang YW, Lin CY, Chang SM, Hsu WL, et al. Prognostic significance of total metabolic tumor volume on. Oncotarget. 2017;8:99587600. doi:10.18632/oncotarget.20447.

25. Oh MY, Chung JS, Song MK, Shin HJ, Lee HS, Lee SM, et al. Prognostic value of Waldeyer's ring involvement of diffuse large B-cell lymphoma treated with R-CHOP. Int J Hematol. 2013;97:397-402. doi:10.1007/s12185-013-1282-3.

26. Song MK, Yang DH, Lee GW, Lim SN, Shin S, Pak KJ, et al. High total metabolic tumor volume in PET/CT predicts worse prognosis in diffuse large B cell lymphoma patients with bone marrow involvement in rituximab era. Leuk Res. 2016;42:1-6. doi:10.1016/j.leukres.2016.01.010.

27. Zwanenburg A, Vallières M, Abdalah MA, Aerts HJWL, Andrearczyk V, Apte A, et al. The Image Biomarker Standardization Initiative: Standardized Quantitative Radiomics for High-Throughput Image-based Phenotyping. Radiology. 2020;295:328-38. doi:10.1148/radiol.2020191145.

28. Aide N, Lasnon C, Veit-Haibach P, Sera T, Sattler B, Boellaard R. EANM/EARL harmonization strategies in PET quantification: from daily practice to multicentre oncological studies. Eur J Nucl Med Mol Imaging. 2017;44:17-31. doi:10.1007/s00259-017-3740-2.

29. Mikhaeel NG, Smith D, Dunn JT, Phillips M, Møller H, Fields PA, et al. Combination of baseline metabolic tumour volume and early response on PET/CT improves progression-free survival prediction in DLBCL. Eur J Nucl Med Mol Imaging. 2016;43:1209-19. doi:10.1007/s00259-016-3315-7.

\section{Figures}




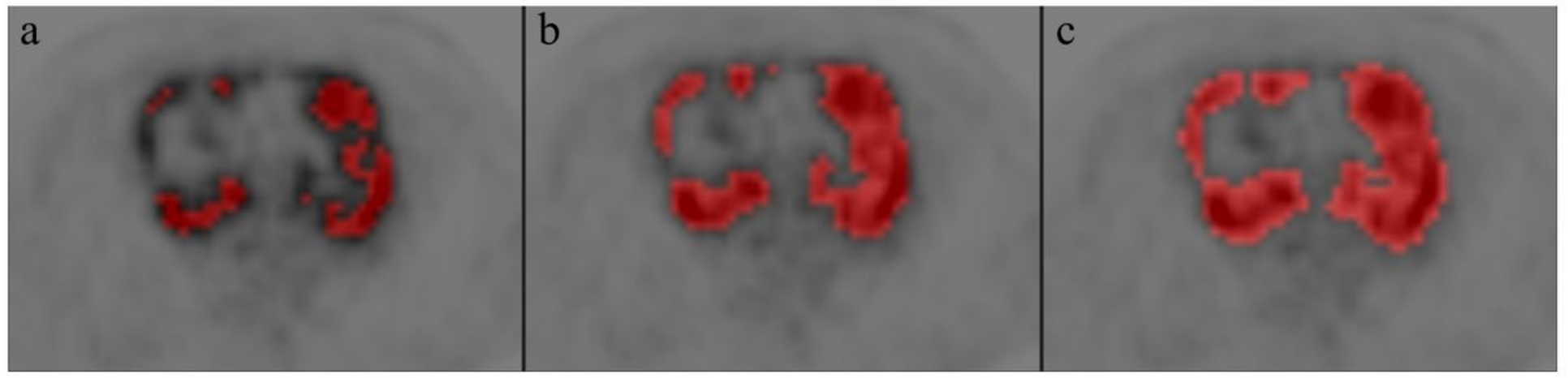

Figure 1

Transaxial PET images with fused mediastinal lymphoma MTV VOls. Different MTV segmentation techniques yielding different MTVs: a) glob4 with $294 \mathrm{~cm} 3$, b) $41 \mathrm{pc}$ with $579 \mathrm{~cm} 3$, and c) grad with $798 \mathrm{~cm} 3$ PET: positron emission tomography; VOI: volume of interest; MTV: metabolic tumour volume; bwaMTV: body weight-adjusted metabolic tumour volume

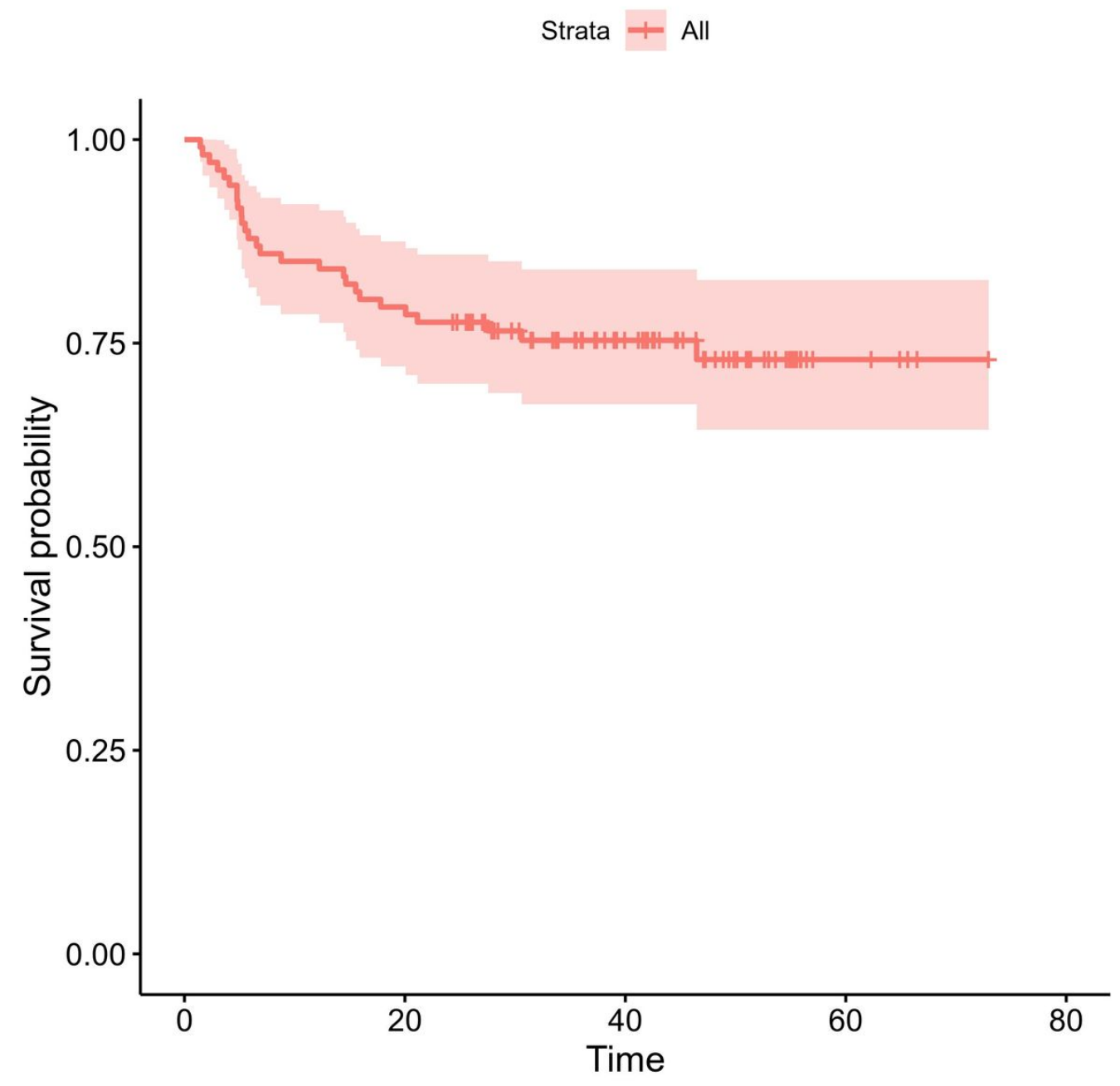

Figure 2

Progression-free survival curve of the patient population 

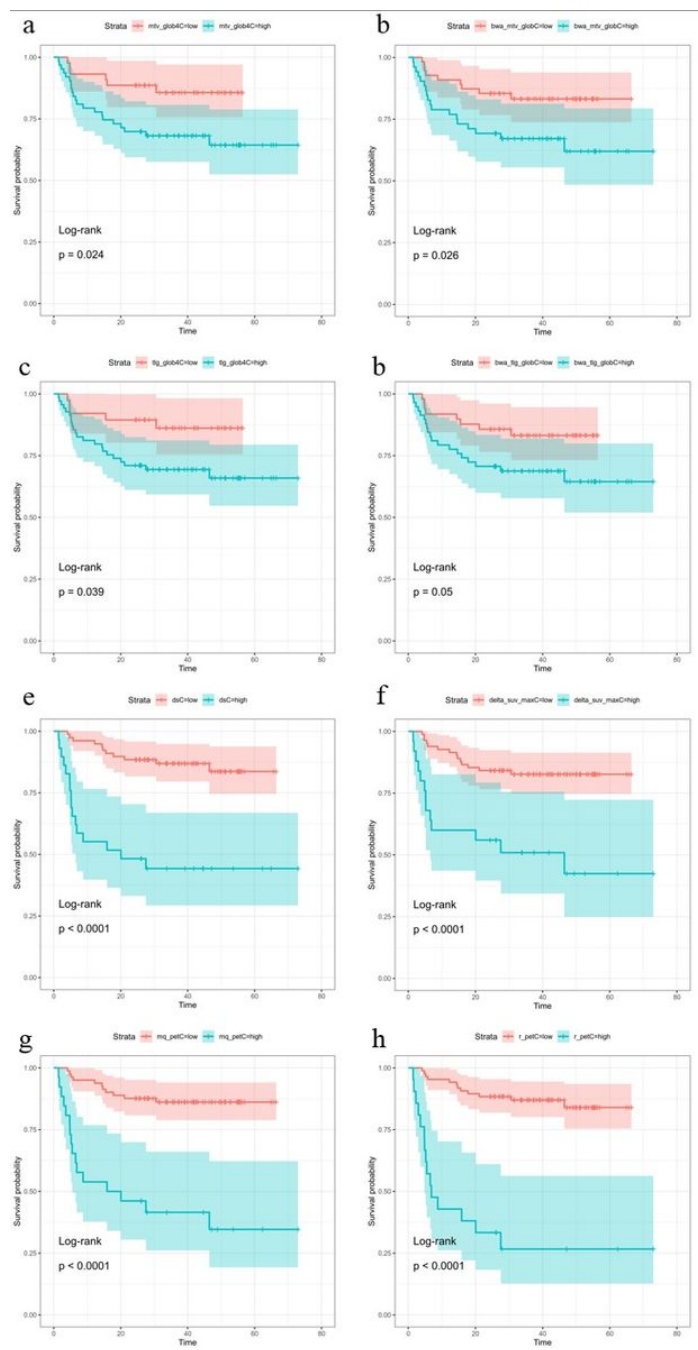

\section{Figure 3}

Kaplan-Meier curves of progression-free survival between low- and high-risk patient groups divided according to baseline MTV (a), bwaMTV (b), TLG (c), bwaTLG (d), and interim parameters DS (e), $\triangle$ SUVmax (f), mqPET (g), and rPET (h) MTV: metabolic tumour volume; TLG: total lesion glycolysis; bwa: body weight-adjusted; DS: Deauville-score; SUVmax: maximum standardized uptake value; mqPET: modified qPET; rPET: ratio PET 


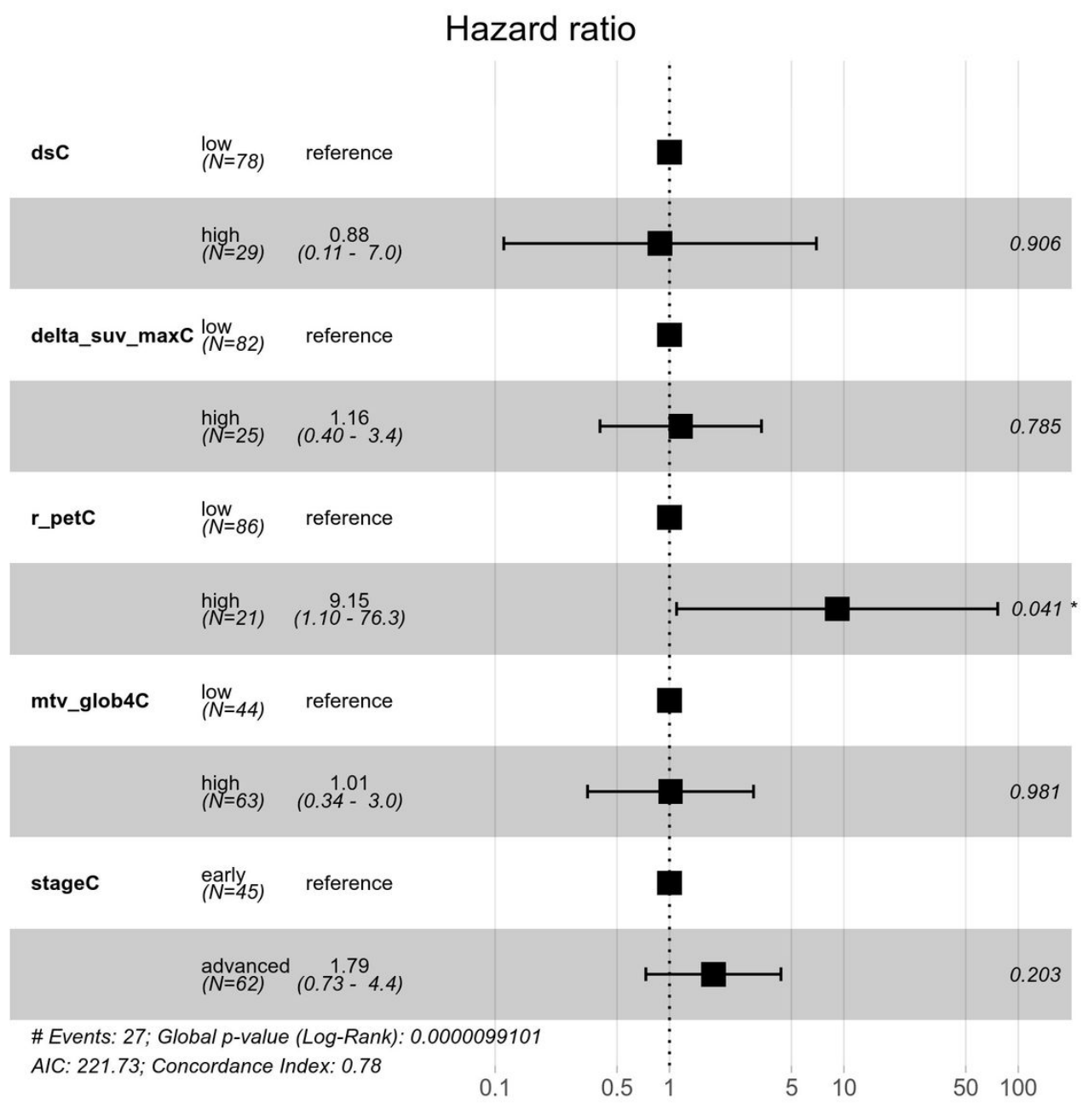

Figure 4

Multivariate Cox-regression model of progression-free survival including Deauville-score, $\triangle$ SUVmax, rPET, MTV, and clinical stage SUVmax: maximum standardized uptake value; rPET: ratio PET; MTV: metabolic tumour volume 
a

Strata + mtv_glob4C=low, dsC=low + mtv_glob4C=low, dsC=high + mtv_glob4C=high, dsC=10w + mtv_glob4C=high, dsC=high
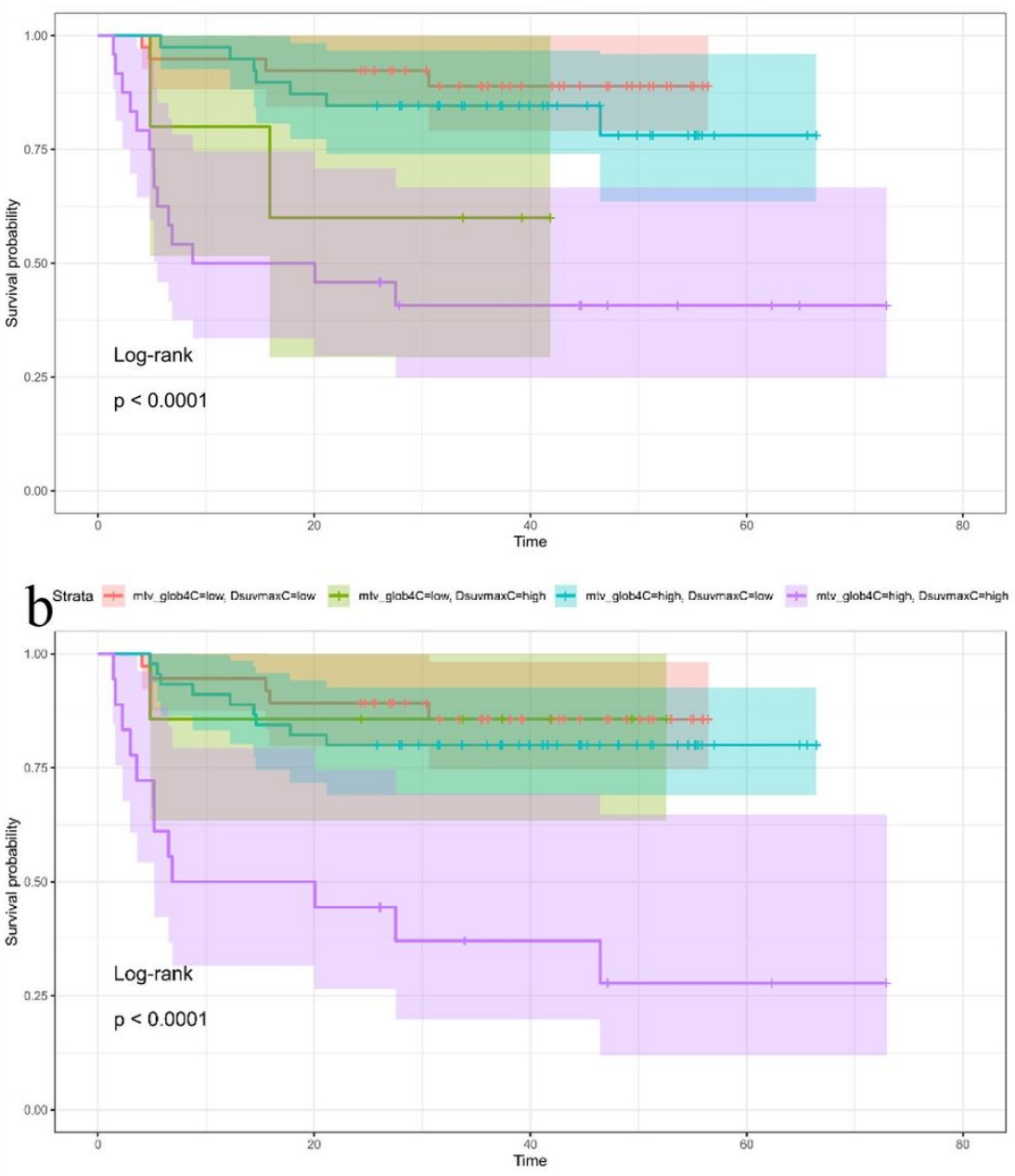

\section{Figure 5}

Kaplan-Meier curves of progression-free survival of a) four subgroups according to low/high MTV and DS 1-3 vs. 4-5 and b) four subgroups according to low/high MTV and low vs. high $\triangle$ SUVmax MTV: metabolic tumour volume; DS: Deauville-score; SUVmax: maximum standardized uptake value 


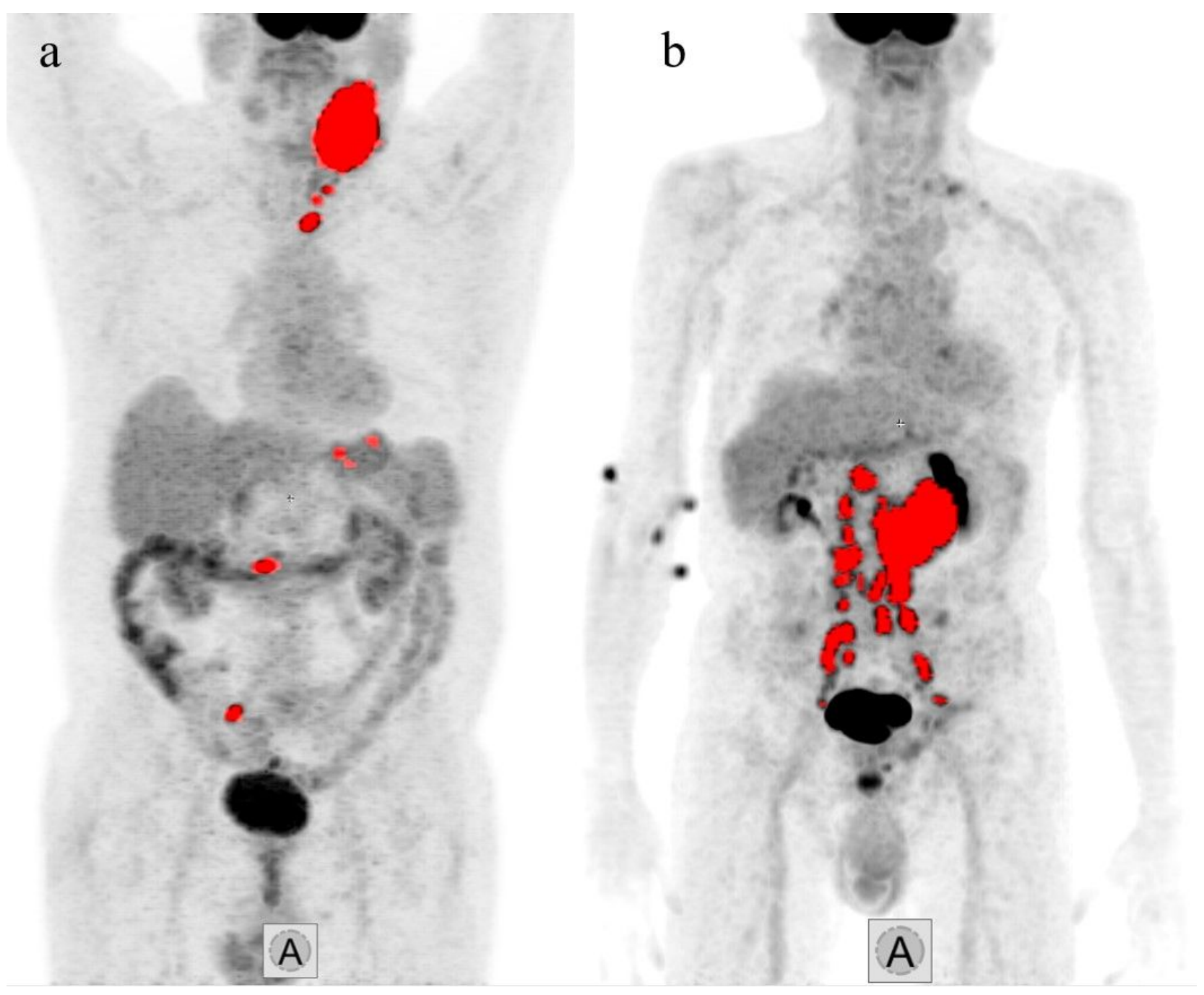

Figure 6

3D MIP PET images with fused MTV VOls. a) $92 \mathrm{~kg}$ patient with MTV of $189 \mathrm{~cm} 3$ and bwaMTV of 1.76 who showed no progression during $51 \mathrm{months}$ of follow-up. b) $54 \mathrm{~kg}$ patient with MTV of $292 \mathrm{~cm} 3$ and bwaMTV of 3.16 who relapsed 7 months after baseline PET (n.b.: radiopharmaceutical skin contamination is present in the right cubital area) MIP: maximum intensity projection; PET: positron emission tomography; VOI: volume of interest; MTV: metabolic tumour volume; bwaMTV: body weight-adjusted metabolic tumour volume 